ACTA

AMAZONICA

\title{
Tres nuevas especies de Righiodrilus Zicsi 1995 (Annelida, Oligochaeta: Glossoscolecidae) de la Amazonía colombiana
}

Alexander FEIJOOํㅗㄴ, Liliana Vanessa Celis GIL²

\section{RESUMEN}

Se describieron tres nuevas especies de Righiodrilus Zicsi, 1995 para la Amazonía: $R$. andake sp. n., R. inga sp. n. y R. muinane. Con las nuevas adiciones el género suma 26 especies. Se presenta una clave para las especies y algunos comentarios relacionados con las características y presencia en usos del terreno de Righiodrilus.

PALABRAS-CLAVE: Región neotropical, Caquetá, Annelida, Lombrices de tierra, Glossoscolecidae.

\section{Três novas espécies de Righiodrilus Zicsi 1995 (Annelida, Oligochaeta: Glossoscolecidae) da Amazônia colombiana}

\section{ABSTRACT}

Three new species of Righiodrilus Zicsi, 1995 from Amazonia were described: $R$. andake sp. n., $R$. inga sp. n. y $R$. muinane sp. $\mathrm{n}$. With these new additions the genus now contains 26 species. A key for species is provided and some remarks related to characteristics and presence of Righiodrilus land uses are furnished.

KEYWORDS: Neotropical region, Caquetá, Annelida, Earthworm, Glossoscolecidae. 


\section{INTRODUCCION}

Entre las contribuciones más recientes sobre la fauna de lombrices de tierra en América Latina se destaca la compilación de Brown \& Fragoso (2007) con registros de la oligoquetofauna, listados de especies, ecología y biogeografía de cada país. En el neotrópico la mayor cantidad de registros se concentran en la Amazonía y en los Andes; pero la colección de lombrices en las tierras bajas amazónicas se ha realizado en sitios aislados de la descomunal ecorregión del Escudo Guyanés, comprendida entre el norte del río Amazonas y el oriente del río Orinoco (Lavelle \& Lapied, 2003; Christoffersen, 2008).

Aunque gran parte de la Amazonía colombiana corresponde a éste Escudo y representa el 30,7\% (315.000 km²) del área total de la nación, hasta el momento no se ha realizado ningún estudio relacionado con taxonomía y ecología de lombrices y sólo se avanzó en el reconocimiento de 130 oligoquetos en la ecorregión del trópico alto-andino y la Orinoquía (Righi, 1995; Feijoo et al., 2004, Feijoo, 2007; Feijoo, 2008).

En el paisaje agroforestal del departamento amazónico del Caquetá domina el uso intensivo en pastizales $(25,7 \%$ de un total de 8.895.600 ha) (Ramírez et al., 2004), ha venido creciendo el caucho (Hevea brasiliensis) en monocultivo o asociado (aproximadamente 5.664 ha). También se observan sistemas arbóreos de frutales, maderables o forrajeros; terrenos abandonados cubiertos por vegetación pionera y sitios de recrecimiento forestal posterior a la extracción de maderas nobles. Este mosaico de paisaje puede ofrecer condiciones favorables o limitantes para las diferentes especies de la oligoquetofauna.

Como buena parte de las identificaciones y descripciones de nuevas especies en ambas ecorregiones fueron realizadas por Gilberto Righi (105 en la amazónica y 36 en la alto Andina), los autores reunieron en este artículo la descripción de tres nuevas especies del género Righiodrilus de las colectadas en la Amazonia colombiana, para hacer un reconocimiento a Gilberto Righi, la persona que más ha contribuido al conocimiento de nuevas especies de lombrices de tierra en América Latina.

\section{MATERIALES Y MÉTODOS}

El paisaje de la Amazonía colombiana muestreado en el presente reconocimiento está constituido por el predominio de fincas dedicadas a la cría de ganadería bovina con siembras de pastos naturales y mejorados, cultivos de caucho (Hevea brasiliensis) y algunos parches con vegetación natural situados en ambientes entre 224 y 302 metros sobre el nivel del mar, con fincas que oscilan entre 8 y 352 ha.

Los animales estudiados se colectaron durante el mes de junio de 2008 en los municipios de Belén de Los Andaquíes (01²1'052”N, 7549'18.8”W y 01²'46.5”N, $\left.75^{\circ} 48^{\prime} 37.8^{\prime \prime} \mathrm{W}\right)$, Florencia (0132'32.5”N, 75³6'02”W y $\left.01^{\circ} 23^{\prime} 31.4^{\prime \prime} \mathrm{N}, 75^{\circ} 29^{\prime} 28.7^{\prime \prime} \mathrm{W}\right)$ y Morelia $\left(01^{\circ} 28^{\prime} 12.4^{\prime \prime} \mathrm{N}\right.$, $75^{\circ} 40^{\prime} 01^{\prime \prime} \mathrm{W}$ y $\left.01^{\circ} 26^{\prime} 24.2^{\prime \prime} \mathrm{N}, 75^{\circ} 42^{\prime} 07^{\prime \prime} \mathrm{W}\right)$ en el departamento del Caquetá, Colombia. Se establecieron 135 puntos de muestreo en 27 fincas y siete categorías de usos del terreno (Pastizal degradado, Pastizal degradado con árboles dispersos, Pastizal mejorado, Cultivo de caucho, Rastrojo, Bosque Intervenido, Sistema Agroforestal).

Las lombrices de tierra fueron extraídas del suelo con dos métodos: TSBF (Anderson \& Ingram, 1993) y con la aplicación de formaldehído al $0,05 \%$ en un área de 0,5 $\mathrm{m}^{2}$ (Raw, 1959), luego se fijaron en formaldehído al 5\% y se conservaron en alcohol al 70\%. El material se examinó en un estereoscopio marca Zeiss (STEMI DV4) mediante observación externa y se utilizaron números arábigos para situar los segmentos, inter segmentos y disepimentos internos, los cuales se enumeraron desde el peristomio hasta el último segmento de la región posterior, se midió la longitud, el diámetro en las regiones preclitelar (segmento 8), clitelar (1820) y posclitelar (35-40) con un calibrador milimétrico. Luego se hizo disección dorsal para observar las estructuras internas, la genitalia, los nefrídios y extraer las cerdas común y genital. Se utilizó un ocular milimetrado con precisión de 0,01 mm para medir la distancia entre las cerdas y establecer la escala de las estructuras con relación al plano de observación. Los holotipos y algunos paratipos se depositaron en la Universidad Tecnológica de Pereira, Colombia y en la colección del Instituto de Ciencias Naturales - Museo de Historia Natural de la Universidad Nacional de Colombia, Sede Bogotá. La construcción de la clave se realizó a partir de los trabajos de Righi (1995) y Zicsi (1995), en algunos casos se conservó las propuestas de ellos y en otros se modificaron o se adicionaron algunas nuevas características.

\section{RESULTADOS}

Género Righiodrilus Zicsi, 1995

Especie tipo: Righiodrilus tico Righi, 1982.

Distribución: Brasil, Colombia, Venezuela.

Diagnosis: Ocho hileras de cerdas estrechamente, en ocasiones, ampliamente pareadas dispuestas en series regulares. Molleja en segmento 6. Un par de poros masculinos intraclitelares. Poros femeninos pares, pocas veces impar. Un par de glándulas calcíferas en el segmento 11 - 12 con estructura tubular compuesta y con membrana traslúcida adicional a la estructura que se prolonga hasta dos o tres segmentos. Aparato genital metándrico y metagínico, con vesículas seminales que ocupan uno o varios segmentos. Espermatecas presentes, rara vez ausentes, usualmente sin cámaras seminales o divertículos. El género se sitúa en la familia Glossoscolecidae por la presencia de molleja en el segmento 6. 


\section{Righiodrilus andake $\mathrm{n} . \mathrm{sp}$.}

Holotipo: Un ejemplar clitelado. Localidad: Belem de los Andaguíes, vereda Aguadulce, 01²0'25.9” N, 75 49'14.9” W, altura sobre el nivel del mar 269 m, 26 de junio de 2008 . Colectores: A. Feijoo, L. Celis. Se depositarán en la colección de la Universidad Tecnológica de Pereira, Colombia.

Paratipos: 12 adultos, siete subadultos, cuatro juveniles. Municipio de Florencia, vereda Alto Canelos, 01 ${ }^{\circ} 28^{\prime} 25.5^{\prime \prime}$ $\mathrm{N}, 75^{\circ} 33^{\prime} 04.5^{\prime}$ 'W, altura sobre el nivel del mar $1135 \mathrm{~m}, 20$ de junio de 2008; Morelia, vereda Cumaral, 01 $266^{\prime} 38.1^{\prime \prime} \mathrm{N}$, $75^{\circ} 41^{\prime} 15.9^{\prime \prime} \mathrm{W}$, altura sobre el nivel del mar $1135 \mathrm{~m}, 24$ de junio de 2008; Belem de los Andaguíes, vereda Agua Dulce, $01^{\circ} 20^{\prime 2} 25.9^{\prime \prime} \mathrm{N}, 75^{\circ} 49^{\prime} 14.9^{\prime \prime}$ W, altura sobre el nivel del mar 1135 m, 26 de junio de 2008. Colectores: A. Feijoo, L. Celis. Se depositarán en la Universidad Tecnológica de Pereira, Colombia y en la colección del Instituto de Ciencias Naturales - Museo de Historia Natural de la Universidad Nacional de Colombia, Sede Bogotá.

Etimología: El nombre de la nueva especie se dio como homenaje y reconocimiento a la comunidad indígena colombiana "Andake" situada en el municipio de Belén de Los Andaquíes, departamento del Caquetá.

Descripción: Especie endogeica despigmentada con coloración blanca lechosa en la región dorso lateral del clitelo. El holotipo entero mide $77 \mathrm{~mm}$ de longitud y tiene 243 segmentos, $0,1 \mathrm{~mm}$ de diámetro en la región preclitelar, 0,15 en el clitelo (segmento 8) y 0,09 mm en la región posclitelar (segmento 40). El prostomio es un pequeño lóbulo dorsal y es poco reconocible en la mayoría de los ejemplares. El clitelo en forma de silla y con fuertes protuberancias que limitan la línea de las cerdas a, ocupa $2.2 \mathrm{~mm}$ de longitud y se sitúa entre los segmentos $1 / 215,15-1 / 223,23$ (= 9 segmentos). En la región ventral se sitúan claramente demarcados y con forma sobresaliente un par de bandas de la pubertad entre los segmentos 17 - 20 (Figura 1A, 1B).

Las cerdas visibles a partir del segmento 3, están dispuestas en ocho hileras ampliamente pareadas y organizadas en series longitudinales regulares. La distancia entre las cerdas en la región media del cuerpo (segmentos $40 \mathrm{al} 50$ ) fue aa: ab: bc: cd: $\mathrm{dd}=3.0: 1.1: 1.0: 1.2: 9.0$. La cerda común presenta una ligera curvatura y en la región distal se engruesa para desembocar en el ápice en una punta pronunciada; mientras que la genital es fuertemente curvada en la región que se inserta en el folículo y luego se proyecta de manera recta hasta terminar en punta con forma de lanza, en el ápice se diferencian seis marcas semilunares que corren alternadas hasta el extremo (Figura 2A, 2B).

Los poros masculinos se sitúan en el intersegmento 18 $\mathrm{y}$ los femeninos aparecen en el segmento 14. Los poros
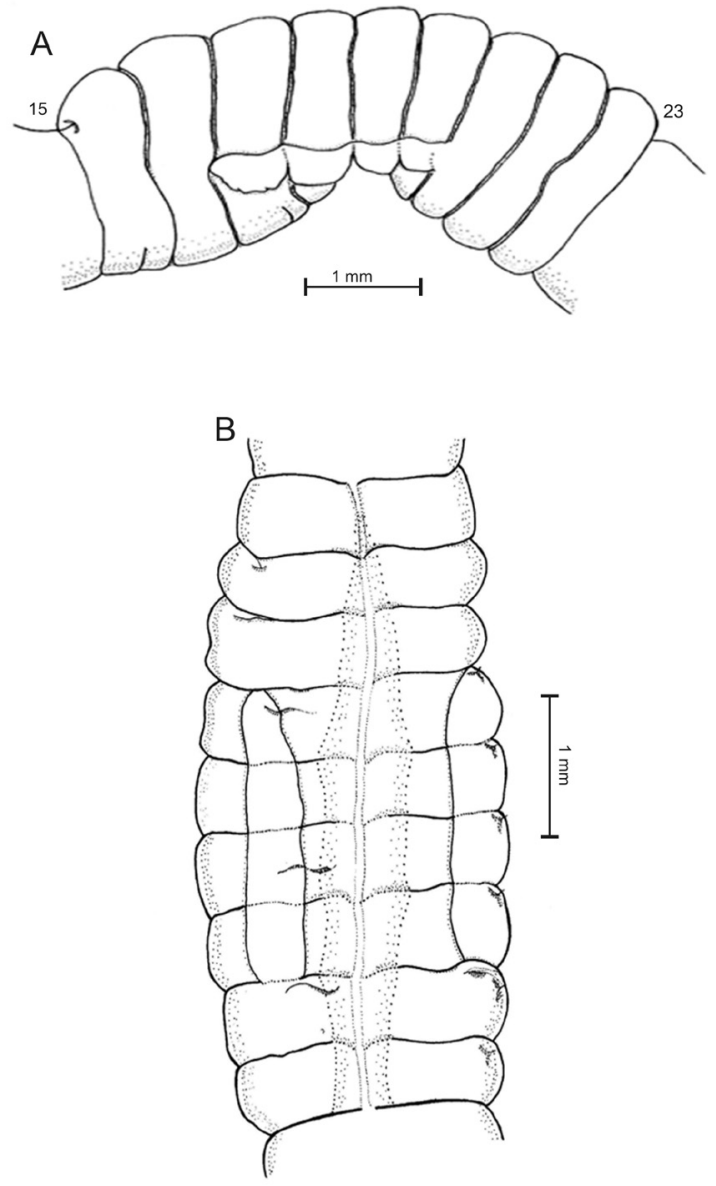

Figura 1 - Righiodrilus andake. A. Vista ventro-lateral del clitelo. B. Vista ventral del clitelo.

espermatecales son visibles en los inter segmentos de $7 / 8$ y 8/9. Los nefridioporos están próximos a la línea de las cerdas b.

Anatomía interna: Los disepimentos entre $7 / 8$ y 10/11 son espesos, gruesos y fuertes con forma cónica y se insertan cerrando en la zona medial del segmento posterior. Una molleja fuerte, muscular y con forma de globo se encontró en la cavidad del segmento 6 . Un par de glándulas calcíferas extramurales con forma de saco alargado y estructura tubular compuesta se encuentra entre los segmentos $11 \mathrm{y}$ 12. La glándula está dividida en dos porciones (glandular y membranosa), la primera con fuerte irrigación de los vasos sanguíneos y túbulos ramificados que parten de dos ramales principales; a partir de $1 / 213$ aparece la segunda con cuatro ramales que forman la base de las membranas. El intestino comienza en el segmento 15 y tiene un tiflosol con forma de lámina delgada en el dorso del interior del intestino que ocupa $1 / 5$ de la luz intestinal. No hay presencia de saco ciego. Se diferencian dos pares de corazones laterales en 7/8/9 y dos pares de corazones intestinales en los segmentos 10 y 11 . Hay un par de holonefrídios por segmento que tienen una vejiga 


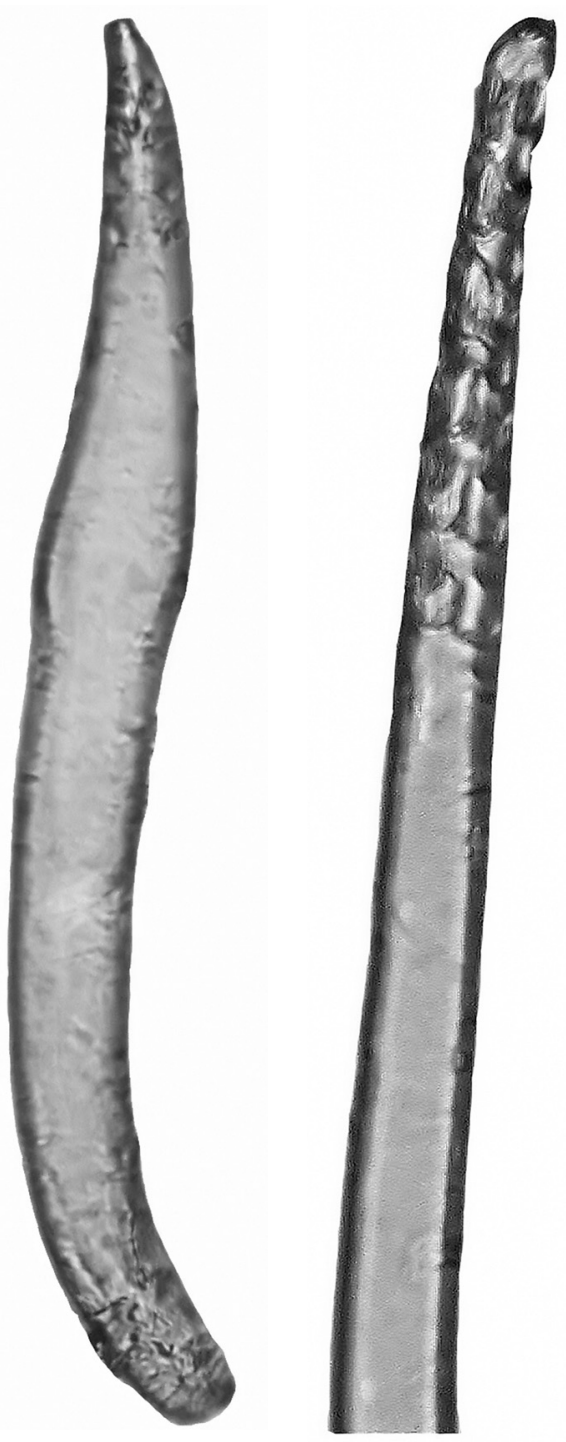

Figura 2 - Righiodrilus andake. A. Seta común, B. Seta genital

delgada (Figura 3A). Dos sacos testiculares que se funden debajo del esófago con color blanco lechoso y abundantes espermatozoides se sitúan en el segmento 11 , los anteriores dan continuidad a un par de vesículas seminales con forma rectangular que se desplazan en la región dorso lateral hasta el segmento 32 (Figura 3B). Un par de conductos corren rectos en la parte superior de la musculatura parietal hasta el centro del segmento 18 en el que desembocan en un par de poros masculinos situados dentro de las bandas de la pubertad. Los conductos masculinos recorren la pared corporal hasta el segmento 17. Hay dos pares de espermatecas llenas de espermatozoides en el segmento $7 / 8$ y 8/9 con un conducto corto y una ámpula redonda, las de $7 / 8$ son de mayor tamaño y con estructura más compleja que las siguientes (Figura 3C).

Consideraciones: $R$. andake sp. n. es una especie cercana a $R$. uete, $R$. aioca, $R$. cigges, $R$. tico, $R$. freitasi debido a
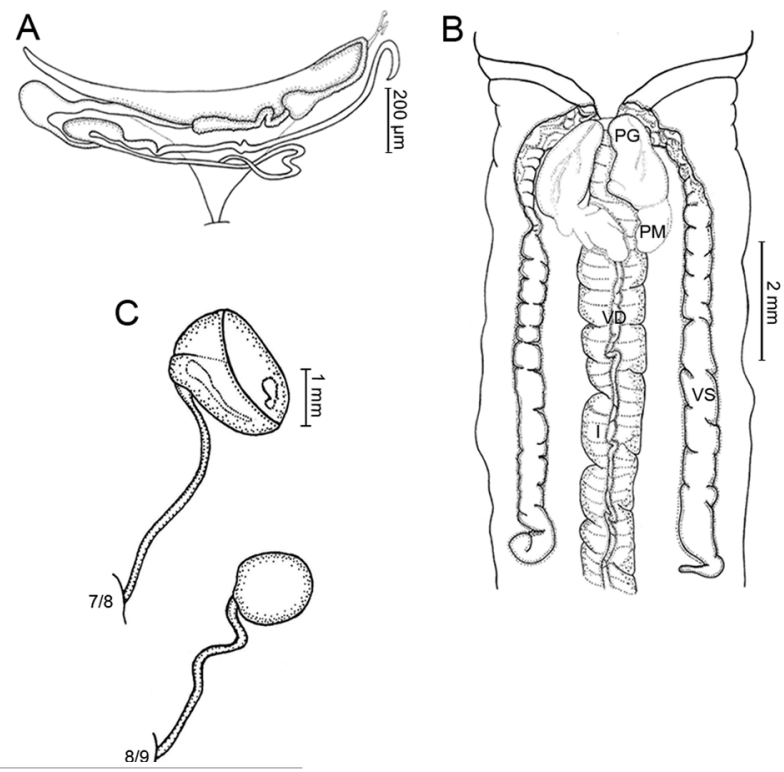

Figura 3 - Righiodrilus andake. A. Nefrídio del segmento 18. B. Aparato genital (PG: Parte glandular de la glándula calcífera, PM: Parte membranosa de la glándula calcífera. VS: Vesículas seminales, I: intestino). C. Espermatecas de $7 / 8$ y $8 / 9$.

la presencia de dos pares de espermatecas en 7/8 - 8/9, se diferencia por tener ocho hileras de cerdas regulares ampliamente pareadas (en las otras se presentan ocho hileras regulares estrechamente pareadas), la ubicación diferente en la distancia en mm de las cerdas aa:ab:bc:cd:dd, la extensión del número de segmentos del clitelo, la extensión del número de segmentos de las bandas de la pubertad y posición de los poros masculinos (Cuadro 1).

\section{Righiodrilus inga n. sp.}

Holotipo: Un ejemplar clitelado. Localidad: Municipio de Florencia, vereda San José Canelo, 01³1'05.5” N, 75³2'50.4” W, altura sobre el nivel del mar 254 m, 22 de junio de 2008. Colectores: A. Feijoo, L. Celis. Se depositarán en la colección de la Universidad Tecnológica de Pereira, Colombia.

Paratipos: Cinco adultos, seis juveniles. Localidad: La misma localidad y fecha de colecta del holotipo. Colectores: A. Feijoo, L. Celis. Se Depositarán en la Universidad Tecnológica de Pereira, Colombia y en la colección del Instituto de Ciencias Naturales - Museo de Historia Natural de la Universidad Nacional de Colombia, Sede Bogotá.

Etimología: El nombre de la especie se dio como homenaje y reconocimiento a la etnia indígena Inga que habita en regiones de los departamentos del Caquetá y Putumayo.

Descripción: Especie endogeica despigmentada. El holotipo entero mide $90,2 \mathrm{~mm}$ de longitud y tiene 226 segmentos, 0,23 mm de diámetro en la región preclitelar, 0,25 en el clitelo (segmento 8) y 0,21 mm en la región posclitelar (segmento 40 ). El prostomio esta invaginado en todos los 
Tres nuevas especies de Righiodrilus Zicsi 1995 (Annelida,

Oligochaeta: Glossoscolecidae) de la Amazonía colombiana

\begin{tabular}{|c|c|c|c|c|c|c|}
\hline Especie & $\begin{array}{l}\text { Longitud } \\
\mathrm{mm} .\end{array}$ & $\begin{array}{l}\text { Número de } \\
\text { segmentos }\end{array}$ & $\begin{array}{l}\text { Distancia de cerdas } \\
\text { aa:ab:bc:cd:dd (mm) }\end{array}$ & $\begin{array}{l}\text { Posición del } \\
\text { Clitelo }\end{array}$ & $\begin{array}{l}\text { Campo genital masculino } \\
\text { (Posición bandas pubertad) }\end{array}$ & $\begin{array}{l}\text { Posición de los } \\
\text { poros masculinos }\end{array}$ \\
\hline R. andake sp. nov. & 77 & 243 & $3,0: 1,1: 1,0: 1,2: 9,0$ & $1 / 215,15-1 / 223,23$ & $\begin{array}{c}\text { Un par de bandas de la } \\
\text { pubertad entre los segmentos } \\
17-20\end{array}$ & 17 \\
\hline R. freitasi & $117-127$ & $276-339$ & $2,6: 1,0: 0,3: 0,2: 2,7$ & $1 / 317-23,24$ & $\begin{array}{c}\text { Dos pares de papilas } \\
\text { confluentes longitudinalmente } \\
\text { en } 19-2 / 323\end{array}$ & $20 / 21$ \\
\hline R. aioca & $\geq 45$ & $\geq 129$ & $1,8: 1,0: 1,0: 0,2: 5,7$ & $16-24$ & $\begin{array}{l}\text { Área rectangular espesa en 1⁄2 } \\
19-22 \text { con un par de surcos } \\
\text { semicirculares en 19/20-21/22. }\end{array}$ & $20 / 21$ \\
\hline R. cigges & $38-45$ & $161-180$ & $2,6: 1,0: 0,8: 0,7: 1,8$ & $15-21$ & $\begin{array}{l}\text { Un par de bandas de la } \\
\text { pubertad en 17-19 }\end{array}$ & $17 / 18$ \\
\hline R. tico & $17-21.5$ & $91-109$ & $2,12: 1,0: 1,04: 0,96: 2.0$ & $16-22$ & $\begin{array}{c}\text { Un par de bandas de la } \\
\text { pubertad en } 1 / 219-1 / 221\end{array}$ & $1 / 219$ \\
\hline R. uete & $70-87$ & $127-145$ & $9,6: 1,0: 3,3: 0,6: 9,6$ & $15-21$ & $\begin{array}{l}\text { Un par de bandas de la } \\
\text { pubertad en } 1 / 219-20\end{array}$ & $19 / 20$ \\
\hline
\end{tabular}

ejemplares. A partir del segmento 8 dos líneas intersegmentales bordean la región ventral y el clitelo en forma de silla aparece entre los segmentos $14-1 / 222$ (= 8,5 segmentos), con fuertes protuberancias que limitan la línea de las cerdas a y ocupa 2,2 mm de longitud. Un par de bandas de la pubertad se sitúan de forma sobresaliente entre los segmentos $1 / 217-20,1 / 220$ (Figura 4A).

Las cerdas a, b, c, d visibles a partir del segundo segmento están dispuestas en ocho hileras estrechamente pareadas y organizadas en series longitudinales regulares. La distancia entre las cerdas en la región media del cuerpo (segmentos 40 al 50) fue aa: ab: bc: $c d: d d=11.8: 0.5: 2.2: 0.4: 14$.

Los poros masculinos se sitúan en el intersegmento 18/19 y los femeninos aparecen en el segmento 14. Los poros espermatecales son visibles en los inter segmentos de 7/8, 8/9, 9/10. Los nefridioporos por encima de las cerdas b.

Anatomía interna: Los disepimentos entre 7/8 - 10/11 gruesos y fuertes con forma cónica. Una molleja fuerte, muscular y con forma de globo se observó en la cavidad del segmento 6. Un par de glándulas calcíferas extramurales con estructura tubular compuesta se sitúa entre los segmentos 11 - 12 con forma de saco alargado que se proyecta hasta el segmento 15 , en 11 y 12 se ubica la parte glandular mientras que la membranosa se extiende entre $13-15$ (Figura 4B). La estructura glandular esta formada por túbulos ramificados, los cuales se abren desde una cavidad central y luego se van entrelazando, la luz del eje central se va ampliando en dirección octal (Figura 4C). La porción membranosa es traslúcida con forma de reservorio que aparece vacío y sólo deja ver pequeńas partículas sin consistencia.

La transición esófago - intestino comienza entre el intersegmento $14 / 15$ y en este último segmento se forma el tiflosol pequeño, recorriendo el dorso hacia atrás en zigzag. No hay presencia de saco ciego. Se diferencian
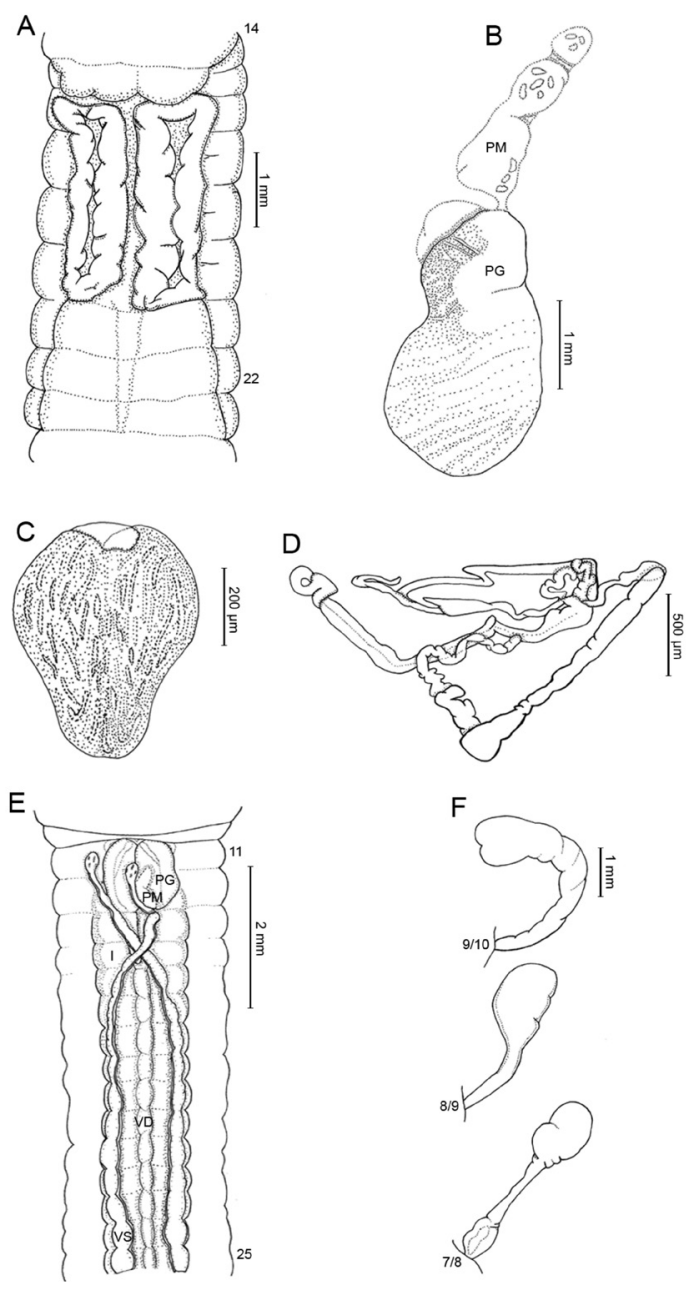

Figura 4 - Righiodrilus inga. A. Vista ventral del clitelo. B. Vista dorsal de la glándula calcífera (PG: Parte glandular de la glándula calcífera. PM: Parte membranosa de la glándula calcífera). C. Corte transversal de la glándula calcífera. D. Nefrídio del segmento 14. E. Aparato genital (PG: Parte glandular de la glándula calcífera, PM: Parte membranosa de la glándula calcífera, VS: Vesículas seminales, I: intestino). F. Espermatecas de 7/8, 8/9 y 9/10. 
tres pares de corazones laterales en 7,8 y 9 y dos pares de corazones intestinales en los segmentos 10 y 11 . Hay un par de holonefrídios por segmento (Figura 4D). Un par de testículos largos se extienden ventralmente en 11. Un par de vesículas seminales parten desde el segmento 12 , se cruzan en el intersegmento 12/13 y se dirigen hacia atrás por la región dorsal hasta el segmento 24, 25; en algunos ejemplares se dirigen hacia atrás por la región ventro lateral cruzándose en el dorso cerca del intestino (Figura 4E).

Los conductos masculinos recorren la pared corporal hasta el segmento 17. Un par de ovarios grandes se diferencian en el segmento 13, cada ovario se presenta como una lámina larga y doblada. Hay tres pares de espermatecas en el segmento $7 / 8,8 / 9,9 / 10$ con un conducto corto y una ámpula redonda (Figura 4F).

Consideraciones: $R$. inga sp. n., es próxima de $R$. tocantinensis, $R$. mucupois, $R$. schubarti y $R$. marcusae por la presencia de tres pares de espermatecas en 7/8, 8/9, 9/10, se diferenció por la ubicación diferente en la distancia en $\mathrm{mm}$ de las cerdas aa:ab:bc:cd:dd, la extensión del número de segmentos del clitelo, la extensión del número de segmentos de las bandas de la pubertad y la posición de los poros masculinos (Cuadro 2).

\section{Righiodrilus muinane $\mathrm{n} . \mathrm{sp}$.}

Holotipo: Un ejemplar clitelado. Localidad: Municipio de Florencia, vereda Alto Canelo, 01²8'19.7” N, 75³2'44.4” W, altura sobre el nivel del mar $252 \mathrm{~m}, 20$ de junio de 2008. Colectores: A. Feijoo, L. Celis. Se depositarán en la Universidad Tecnológica de Pereira, Colombia.

Paratipos: Tres adultos decapitados en la región posterior, un subadulto, un juvenil; la misma localidad y fecha de colecta del holotipo. Colectores: A. Feijoo, L. Celis. Se depositarán en la Universidad Tecnológica de Pereira, Colombia y en la colección del Instituto de Ciencias Naturales - Museo de Historia Natural de la Universidad Nacional de Colombia, Sede Bogotá.

Etimología: El nombre de la especie se dio como homenaje y reconocimiento a la etnia indígena Muinane que habita en algunas áreas del departamento del Caquetá.

Descripción: Especie endogeica despigmentada. El holotipo tiene una longitud de $34 \mathrm{~mm}, 122$ segmentos, mide en el diámetro preclitelar 0,08 mm, clitelar 0,08 (segmento 8) y posclitelar 0,07 (segmento 38). El prostomio es alargado en forma de bastón y sólo es posible visualizarlo en los ejemplares disectados. El clitelo anular se ubica entre los segmentos 14 - 21 (=8 segmentos), con color blanco lechoso se diferencia del resto del cuerpo (Figura 5A). En la región ventral del segmento 17 se encuentran las marcas genitales (poroforos masculinos), próximo a línea de las cerdas ab con forma de papilas cónicas ligadas por una línea transversal que las une en la parte superior. Las papilas tienen color blanco lechoso y en el ápice hay una proyección que le da fin a la estructura con forma de tetilla.

Las cerdas visibles a partir del segmento 3 están dispuestas en ocho hileras estrechamente pareadas y organizadas en series longitudinales regulares. La distancia entre las cerdas en la región media del cuerpo (segmentos 40 al 50) fue aa: ab: bc: $c d: \mathrm{dd}=4.0: 0.2: 2.0: 0.2: 10$. La cerda común es recta en el extremo apical presenta un leve engrosamiento (Figura 5B). La cerda genital no fue posible visualizarla.

Los poros masculinos se sitúan en el segmento 17 y los femeninos aparecen en el segmento 14. Los poros de las

Cuadro 2 - Especies de Rhighiodrilus con tres pares de espermatecas en 7/8, 8/9, 9/10

\begin{tabular}{|c|c|c|c|c|c|c|}
\hline Especies & $\begin{array}{l}\text { Longitud } \\
\mathrm{mm} \text {. }\end{array}$ & $\begin{array}{l}\text { Número de } \\
\text { segmentos }\end{array}$ & $\begin{array}{l}\text { Distancia de cerdas } \\
\text { aa:ab:bc:cd:dd (mm) }\end{array}$ & $\begin{array}{l}\text { Posición del } \\
\text { Clitelo }\end{array}$ & $\begin{array}{l}\text { Campo genital masculino } \\
\text { (Posición bandas pubertad) }\end{array}$ & $\begin{array}{l}\text { Posición de los } \\
\text { poros masculinos }\end{array}$ \\
\hline R. inga sp. nov. & 90.2 & 226 & 11.8: $0.5: 2.2: 0.4: 14$ & $14-1 / 222$ & $\begin{array}{c}\text { Un par de bandas de la } \\
\text { pubertad entre segmentos } 1 / 2 \\
17-20,1 / 220\end{array}$ & 17 \\
\hline R. marcusae & 112 & ---- & ------------------------ & $1 / 213-22$ & $\begin{array}{l}\text { Un par de bandas de la pubertad } \\
\text { entre segmentos } 2 / 317-20 \mathrm{y} \\
\text { un par de papilas en } 14\end{array}$ & 18 \\
\hline R. mucupois & $43-48$ & $161-185$ & $2,4: 1,0: 0,9: 0,6: 1,8$ & $1 / 215-21$ & $\begin{array}{c}\text { un par de bandas de la } \\
\text { pubertad entre los segmentos } \\
1 / 3,1 / 216-19\end{array}$ & $16 / 17$ \\
\hline R. schubarti & 165 & 260 & 13,9: 1,0: 2,4:0,9: 17,6 & $15-24$ & $\begin{array}{c}\text { un par de bandas de la } \\
\text { pubertad entre los segmentos } \\
1 / 317,18-1 / 223 \text { y un par de } \\
\text { papilas en } 16 \text { y } 17\end{array}$ & 18 \\
\hline R. tocantinensis & 132 & 232 & 79: 4: 13: 3.5: 73: 96 & $15-22$ & $\begin{array}{c}\text { un par de bandas de la } \\
\text { pubertad entre los segmentos } \\
1 / 217-1 / 220 \text { y Papila media } \\
\text { ventral en } 21\end{array}$ & 18 \\
\hline
\end{tabular}



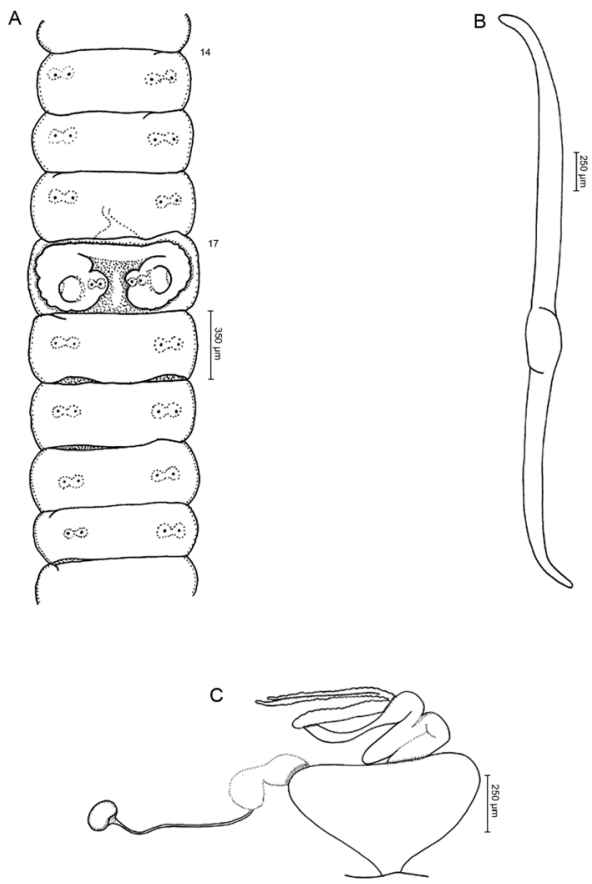

Figura 5 - Righiodrilus muinane. A. Vista ventral del clitelo. B. Seta común. C. Nefrídio del segmento 18.

espermatecas están ausentes. Los nefridioporos están próximos a la línea de las cerdas cd.

Anatomía interna: Los disepimentos entre 7/8 y 10/11 son espesos, gruesos y fuertes con forma de embudo. Una molleja fuerte, muscular y con forma de globo se encontró en la cavidad del segmento 6. Un par de glándulas calcíferas extramurales se visualizan en los segmentos $11-12$, son de estructura tubular compuesta y aparecen debajo de los testículos y vesículas seminales.

El intestino comienza en el segmento 17 y el tiflosol aparece desde el 19 con forma de lámina delgada en $S$ que ocupa $1 / 5$ del diámetro intestinal. No hay presencia de saco ciego. Se diferencian tres pares de corazones laterales en 7, 8 y 9 y dos pares de corazones intestinales en los segmentos 10 y 11. Hay un par de holonefrídios por segmento que tienen un infundíbulo simple al cual se conecta por medio de un largo conducto y termina en el nefrostoma (Figura 5C). Un par de testículos se encuentran en el segmento 11. Un par de vesículas seminales cortas en el segmento 12 . Los conductos de color blanquecino parten desde el segmento 11 y se extienden en forma de zigzag hasta el segmento 17 donde desembocan en los poros masculinos. Un par de ovarios se observan en la región posterior ventral del segmento 13 . No hay presencia de espermatecas.

Consideraciones: De las 26 especies del género sólo en Righiodrilus venancioi y $R$. muinane faltan las espermatecas y presentan vesículas seminales cortas; las dos especies se diferencian por las siguientes características: $R$. venancioi presenta poroforo masculino con un par de tumescencias ovoides en línea con las cerdas ventrales (entre 1/3 16-2/3 17 ), mientras que en $R$. muinane los poroforos se presentan sólo en el segmento 17; también se diferencian por la extensión del clitelo (15-22 en forma de silla en $R$. muinane y 14-21 anular en $R$. venancioi) y el inicio del intestino (15 en $R$. muinane y 17 en $R$. venancioi).

\section{DISCUSIÓN}

Los géneros Righiodrilus y Glossodrilus son de tamaño pequeño (25 a 90 mm de longitud) excepto $R$. oliveirae (180 $250 \mathrm{~mm}$ ) y $R$. amazonius $(140 \mathrm{~mm}$ ), en la mayoría de los casos carecen de pigmentación y el color generalmente se los confiere el contenido intestinal, son endógeicas y se encuentran en el suelo entre 5 y $60 \mathrm{~cm}$ de profundidad, carecen de cámaras copulatorias y tienen un solo par de testículos en el segmento 11 (metándricas). El género Righiodrilus se ha encontrado en selvas húmedas neotropicales, Glossodrilus por encima de los 1000 metros de altura sobre el nivel del mar. En Righiodrilus se presenta además una membrana adicional con forma de saco (reservorio) en las glándulas calcíferas $(11-12)$, que en la mayoría de las especies atraviesa los disepimentos posteriores llegando hasta el segmento 16 ( $R$. andake, $R$. inga, $R$. paolettii, $R$. schubarti, $R$. venancioi).

Las especies del género se diferencian por la ausencia de espermatecas ( $R$. muinane, $R$. venancioi), o por la presencia de uno ( $R$. guabibo, $R$. uete dithecae), dos ( $R$. amazonius $)$, tres $(R$. tinga, $R$. schubarti) o seis pares de espermatecas ( $R$. sucunduris). En la mayoría de los casos las vesículas seminales son largas, en otros casos son cortas y restringidas al segmento $12(R$. muinane), en algunas especies son longitudinales y divididas en dos ( $R$. tico, $R$. ortonae, $R$. oliveirae) o tres ramales $(R$. schubartii) (Righi, 1995).

$R$. andake fue la especie con mayor abundancia (1 y 18 individuos $/ \mathrm{m}^{2}$ ) y amplia capacidad de adaptación a ambientes con barbechos o sucesiones naturales de vegetación (Piperaceae, Melastomataceae, Cecropiaceae), perturbados (pastizales degradados con Paspalum notatum o mejorados con Brachiaria decumbens o $B$. brizantha) y en sistemas agroforestales con plantaciones de $H$. brasiliensis asociadas con árboles frutales (Teobroma grandiflorum, T. cacao, Annona sp., Eugenia stipitata, Borojoa patinoi, Bactris gasipaes), y maderables (Cariniana pyriformis, Lecytis sp., Cordia alliodora, Jacaranda copaia). En $R$. inga la abundancia fue baja (entre 1 y 7 individuos $/ \mathrm{m}^{2}$ ) y se encontró en pastizales degradados y mejorados, mientras que $R$. muinane sólo se halló en un muestreo $\left(6\right.$ individuos $/ \mathrm{m}^{2}$ ) en barbechos.

Las variables condiciones para las dinámicas de las comunidades de lombrices que ofrecen los paisajes antropogénicos en la Amazonía colombiana, se pueden deducir a partir de la presencia y abundancia de las tres especies 
de Righiodrilus. $R$. andake parece tener mayor capacidad de adaptación ya que se encontró en diferentes ambientes transformados tales como herbáceos, herbáceos con arbustos y arbóreos. La tolerancia de hábitat en $R$. inga parece más limitada pues solo se encontró en paisajes herbáceos. $R$. muinane puede recolonizar los paisajes transformados cuando ha avanzado el crecimiento del rastrojo en el barbecho.

\section{CLAVE PARA LAS ESPECIES DE RIGHIODRILUS}

1. Espermatecas ausentes..... 2

- Espermatecas presentes. 3

2 Campo genital masculino con poroforos en el segmento 17. R. muinane $\mathrm{n}$. $\mathrm{sp}$.

- Campo genital masculino con un par de tubérculos de la pubertad en la región anterior de 17 o en medio de $1 / 216$ $-1 / 217$. R. venancioi (Righi, 1982)

3 Seis pares de espermatecas en 8/9-10/11.... R. sucunduris (Righi et al., 1976)

- Tres pares de espermatecas en 6/7 8/9.......................................... R. tinga (Righi, 1971)

- Tres pares de espermatecas en 7/8-9/10 ..................... 7

- $\quad$ Dos pares de espermatecas en 7/8-8/9 ........................... 4

- Dos pares de espermatecas en 9/10-10/11......... R. amazonius (Zicsi \& Csuzdi,1999)

- Dos pares de espermatecas en 8/9-9/10 ..16

- Un par de espermatecas en 8/9... R. dithecae (Righi, 1988)

- Un par de espermatecas en $9 / 10$. R. guabibo (Righi \& Molina, 1994)

4 Bandas de la pubertad en $1 / \mathrm{n} 17-1 / \mathrm{n}$ 19............................. cigges (Righi, 1970)

- Bandas de la pubertad $1 / 219-1 / 221 \ldots \ldots \ldots \ldots \ldots \ldots \ldots \ldots . . .5$

- Bandas de la pubertad $1 / 217-1 / 220 \ldots \ldots \ldots \ldots \ldots \ldots \ldots . . . .8$

- Bandas de la pubertad en 16,1/2 16-1/n 18,1/2 19-20.......... 11

- Campo de la pubertad en forma cuadrangular en los segmentos 19-22, con un par de surcos semicirculares en $19 / 20-21 / 22$

... 6

- Papilas de la pubertad ausentes en 21. Surcos de la pubertad con colores oscuros en 18, 19-20.

.R. tocantinensis pola (Righi, 1984)

- Poros masculinos en 18. Papila media ventral en 21. Papilas de la pubertad presentes en el campo genital $1 / 217-1 / 2$ 20............ R. tocantinensis tocantinensis (Righi, 1972)

- Dos pares de papilas confluentes longitudinalmente en 19$2 / 323$ R. freitasi (Righi, 1971)

5. Clitelo en $16-22$. Poros masculinos in $1 / 219$ R. tico (Righi, 1982)

6. Clitelo en 16-24. R. aioca (Righi, 1975)
Clitelo en 1/2 15, 15 - 1/2 23, 23. Bandas de la pubertad den 17 - 20. Poros espermatecales en $7 / 8$ y

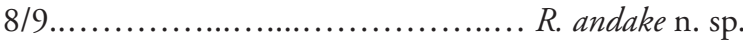

- Clitelo anular en los segmentos $15-21$..................... 9

7. Clitelo en $15-24$........ R. schubarti (Righi et al., 1978)

8. Clitelo en $13-22$. Un par de papilas de la pubertad en 14 ..... R. marcusae (Righi, 1969)

- Clitelo en $1 / 2$ 15-21 .10

9. Poros masculinos en $19 / 20$. Circundantes y estrechas bandas de la pubertad en $1 / 2$ 19-20, sin surco medio longitudinal. En la región media del cuerpo $\mathrm{aa}=\mathrm{dd}=10 \mathrm{ab}$. R. uete (Righi, 1988)

Poros masculinos en 19/20. Clitelo en los segmentos $1 / 2$ $16-22$. Bandas de la pubertad $1 / 218-1 / 220$. Dos pares de espermatecas en 8/9-9/10 ....... $R$. arapaco (Righi, 1982)

10. En la región media aa $<3$ ab.......R. mucupois (Righi, 1970)

11. Cerdas en forma bicúspide. Bandas de la pubertad en $1 / 216$ - 18. Espermatecas en línea con las cerdas b. . .12

Cerdas en forma bicúspide. Bandas de la pubertad en $1 / 216-1 / 2$ 18. Espermatecas en línea con las cerdas d R. fontebonensis (Righi, 1988)

12 Sacos testiculares ausentes. Testículos e infundíbulos masculinos libres. Cerdas bicúspides o bifurcadas R. itajo (Righi, 1971)

13 Bandas de la pubertad en 1/n 17-1/n18 .14

- Bandas de la pubertad en 17- 1/2 20 con línea media longitudinal en $\mathrm{ab}$ 15

14 Vesículas seminales divididas en dos ramales. La forma de las espermatecas No diferencia entre el conducto y la ámpula. ......................... R. ortonae (Righi, 1988) Un par vesículas seminales parten de 12 hasta segmento 24, 25. Tres pares de espermatecas en 7/8-9/10, con un conducto corto y una ámpula redonda..... $R$. inga $\mathrm{n}$. sp.

15 Clitelo en 15-21.............. R. paolettii (Righi, 1984)

- Clitelo en 15-24. Tamańo entre 180-250 mm de longitud... R. oliveirae (Righi, 1982)

16 Bandas de la pubertad amplias en 19-1/321. Con área central un poco más oscura que el cuerpo. La región media del cuerpo $\mathrm{aa}=15.5, \mathrm{dd}=23 \mathrm{ab} \ldots \ldots \ldots \ldots \ldots . . . . . R$. mairaro $($ Righi, 1982)

\section{AGRADECIMIENTOS}

A Erika L. Gordillo y Bertha L. Ramírez de la Universidad de la Amazonía (Caquetá) por la colaboración durante la ejecución del proyecto AMAZ_BD. A María Constanza Zúñiga T., Mónica Díaz P., Andrés F. Carvajal, Darío Ruiz y Sebastián Ramírez miembros del grupo Gestión en Agroecosistemas Tropicales Andinos (GATA) de la 
Universidad Tecnológica de Pereira por su acompańamiento participación en el proyecto. Al maestro Heimar Quintero V. de la Universidad Nacional de Colombia por los aportes y comentarios al documento. Patrick Lavelle (IRD, Francia) por aportes durante la coordinación del proyecto "Biodiversité des paysages Amazoniens. Déterminants socio-économiques et production de biens et services écosystémiques" (AMAZ_BD). A la Agence Nationale de la Recherche (ANR) y L'institut Français de la Biodiversité (IFB) por la financiación del proyecto. A los dos evaluadores anónimos por las sugerencias y contribuciones al manuscrito.

\section{REFERENCIAS BIBLIOGRÁFICAS}

Anderson, J.M.; Ingram, J.S.I. 1993. Tropical Soil Biology and Fertility: a Handbook of Methods. CAB International, Oxford. 221 pp.

Brown, G.; Fragoso, C. 2007. Minhocas na América Latina: Biodiversidad e Ecologia. EMBRAPA Soja, Londrina, Brasil. 545pp.

Cognetti de Martiis L. 1905. Oligocheti raccolti nel Darien dal Dr. Festa E. Boll. Musei Zool. Anat. Comp. R. Univ. Torino, 20: 1-7.

Christoffersen, M. L. 2008. Diversity and distribution of Aptodrilus, Glossodrilus, Onoreodrilus and Righiodrilus (Annelida, Clitellata, Lumbricina, Glossoscolecidae) in South America. Gaia Scientia 2: $21-30$.

Feijoo, A. 2007. Registros históricos y listado de las lombrices de tierra de Colombia, p. 141-153. In: Brown, G.G.; Fragoso, C. (Eds.), Minhocas na América Latina: Biodiversidade e ecologia. Embrapa Soja, Londrina. Embrapa.

Feijoo, M. A. 2008. Lombrices de tierra (Annelida, Oligochaeta) del Parque Nacional Sumapáz, Colombia. In: van der Hammen (Ed.). Studies on Tropical Andean Ecosystems v. 7. J. Cramer, Berlin.

Feijoo, M. A; Quintero, H.; Fragoso, C.; Moreno, G. A. 2004. Patrón de distribución y listado de especies de las lombrices de tierra (Annelida, Oligochaeta) en Colombia. Acta Zoológica Mexicana, 20: 197-220.

Lavelle, P.; Lapied, E. 2003. Endangered earthworms of Amazonia: an homage to Gilberto Righi. Pedobiol, 47: 419-427.

Michaelsen, W. 1927. Oligochätenfauna brasiliens. Abhandl. Senckenberg. Naturforsch. Ge, 40: 369-374.

Ramírez, B.; Estrada, C. A.; Rodríguez, J. G.; Muñoz, J; Guayara, A. 2004. Aporte al conocimiento y sostenibilidad del agroecosistema intervenido de la Amazonia colombiana. Feriva S.A, Cali, Colombia. 221pp.
Raw, F. 1959. Estimating earthworm populations by using formaling. Nature, 184: 1661-1662.

Righi, G. 1969. On some Brazilian Glossoscolecidae. Beiträge zur Neotropischen. Fauna, 6: 152-162.

Righi, G. 1970. Sobre o genero Andioscolex (Oligochaeta, Glososcolecidae). Revista Brasileira de Biologia, 30: 371-376.

Righi, G. 1971. Sobre a família Glossoscolecidae (Oligochaeta) no Brasil. Arquivos de Zoologia., 20: 1-96.

Righi, G. 1972. Contribuição ao conhecimento dos Oligochaeta brasileiros. Papéis Avulsos de Zoologia, 25: 149-166.

Righi, G. 1975. Some Oligochaeta from the Braziliam Amazonia. Studies on Neotropical Fauna and Environ, 10: 77-95.

Righi, G. 1982. Oligochaeta, Glossoscolecidae, do Parque Nacional da Amazonia, Tapajós. Revista Brasileira de Biologia, 42: 107-116.

Righi, G. 1984. Nova Contribuição ao conhecimento dos oligochaeta da Venezuela. Papéis Avulsos de Zoologia, 35(22): 243-256.

Righi, G. 1988. Uma coleção de Oligochaeta da Amazônia Brasileira. Papéis Avulsos de Zoologia, 36: 337-351.

Righi, G. 1995. Colombian earthworms, p. 485-607. In: van der Hammen, T.; Santos, A.G. (Eds). Studies on tropical Andean Ecosystems 4. Berlín: Cramer.

Righi, G.; Ayres, I. 1975 Alguns Oligochaeta sul brasileiros. Revista Brasileira de Biologia, 35: 309-316.

Righi, G.; Ayres, I.; Bittencourt, E. 1976. Glossoscolecidae (Oligochaeta) do Instituto Nacional de Pesquisas da Amazônia. Acta Amazonica, 6: 335-367.

Righi, G.; Ayres, I.; Bittencourt, E. 1978. Oligochaeta (Annelida) do Instituto Nacional de Pesquisas da Amazônia. Acta Amazonica, 8 (3), supl. 1: 1-49.

Righi, G.; Molina, S. 1994. Terricolous Oligochaeta (Glossoscolecidae) of the Venezuela Latinos. Revue Suisse de Zoologie, 101: 299313.

Zicsi, A. 1995. Revision der Gattun Glossodrilus Cognetti, 1905 auf Grund der Arten aus dem Andengebiet (Oligochaeta: Glossoscolecidae) regenwürmer aus sudamérica, 25. Opuscula Zoologica, 27-28: 79-116.

Zicsi, A.; Csuzdi, C. 1999. Neue und bekannte Regenwürmer aus verschiedenen Teilen Südamerikas (Annelida, Oligochaeta: Glossoscolecidae, Acanthodrilidae, Megascolecidae). Senckenbergiana biologica., 78: 123-134.

Recebido em 30/07/2009

Aceito em 16/10/2009 
